\title{
TREINAMENTO DE FORÇA NO \\ RETARDO DO PROCESSO DE \\ SARCOPENIA EM IDOSOS
}

DOI: $10.48140 /$ digitaleditora.2020.002.4

\section{4}

RESUMO

Objetivos: A presente pesquisa possui como objetivo analisar os benefícios do treinamento de força no retardo do processo de sarcopenia em pessoas da terceira idade.

Métodos: Para fundamentação da pesquisa a metodologia é caracterizada como uma revisão bibliográfica ou literária, analisando a teoria referente a temática.

Resultados: Constata-se por meio da literatura que o treinamento de força é um exercício de suma importância em prol do retardo da sarcopenia em idosos.

Conclusão: Salienta-se que a pesquisa pertinente, constatando que o treinamento de força pode contribuir significativamente para que ocorra um retardo no processo de sarcopenia em pessoas idosas.
Narginara Bruna Meneses da

Silva

Graduanda em Educação Física pela AESPI - Ensino Superior do

Piauí - Teresina - Piauí

iD https://orcid.org/00000003-2315-3395

Rainara do Nascimento Morais Graduanda em Educação Física pela AESPI - Ensino Superior do Piauí

Teresina - Piauí

iD https://orcid.org/00000002-0740-2067

Diego Rodrigues Pessoa

Fisioterapeuta, Mestre e Professor Assistente da AESPI - Ensino Superior do Piauí

Teresina - Piauí

https://orcid.org/0000 0002-8981-673X 


\section{STRENGTH TRAINING IN DELAYING THE SARCOPENY PROCESS IN ELDERLY}

DOI: 10.48140/digitaleditora.2020.002.4

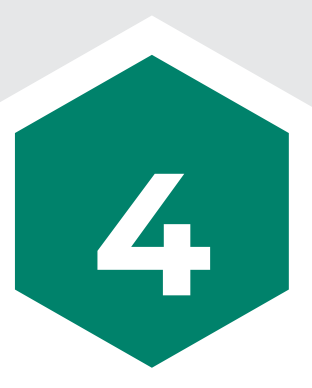

ABSTRACT

Recebido em: 10/12/2020

Aprovado em: 28/12/2020

Conflito de Interesse: não

Suporte Financeiro: não houve

Objectives: The present research has as objective to analyze the benefits of strength training in delaying the sarcopenia process.

Methods: To support the research, the methodology is characterized as a bibliographic or literary review, analyzing the theory related to the theme.

Results: It appears through the literature that strength training is an exercise of paramount importance in favor of delaying sarcopenia in the elderly.

Conclusion: It should be noted that the relevant research, noting that strength training can significantly contribute to delaying the sarcopenia process in the elderly. 


\section{(8)}

\section{INTRODUÇÃO}

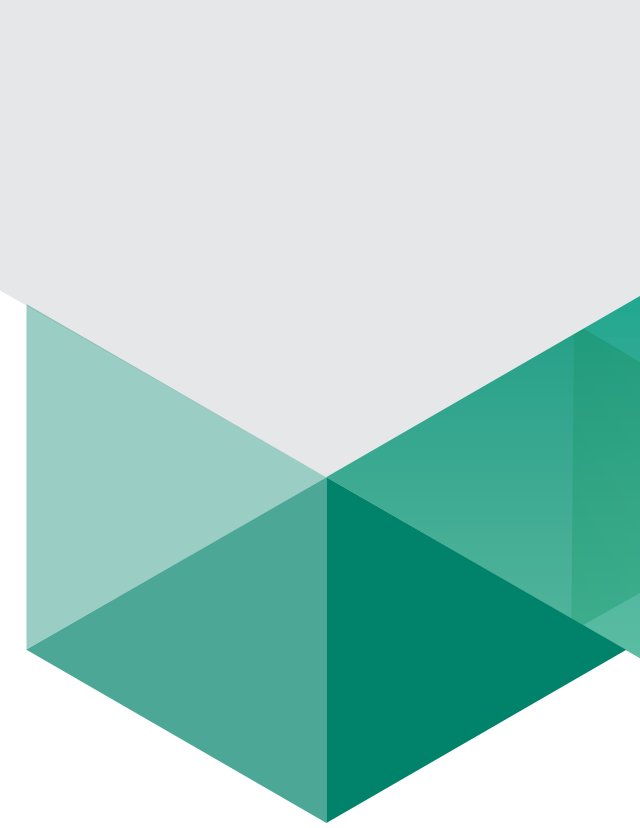

À proporção que há o aumento do número de idosos, em esfera mundial, também se ampliam as discussões referentes a eventos incapacitantes na idade igual ou superior a 60 anos, ressaltando, principalmente, a incidência de quedas entre a população idosa, pois com o processo natural de envelhecimento do corpo humano, há consequentemente, a diminuição da flexibilidade nas articulações e da força dos músculos, impondo, dessa forma, uma série de limitações na qualidade de vida dessas pessoas da terceira idade (STEFFL, 2016).

O processo de envelhecimento imprime várias transformações de ordem natural em todo o organismo do ser humano, havendo uma progressiva e contínua atrofia nos músculos, causando, dentre outros malefícios a fraqueza funcional, a perda de cálcio nos ossos, ampliação da espessura da parede de vasos, crescimento significativo do nível de gordura total no corpo e redução considerável de aspectos relacionados com a capacidade de coordenação, causando, assim, uma maior vulnerabilidade na execução de ações cotidianas (PAGOTTO, 2014).

Para que ocorra uma espécie de retardo das consequências do processo natural de envelhecimento há a necessidade de uma maior estimulação corporal global, em prol do favorecimento de melhorias no desempenho das atividades cotidianas. Dessa forma, infere-se que existem várias alternativas para que aqueles que estão ingressando na faixa etária referente a terceira idade a realização de programas de atividades físicas de treino aeróbicos, treinos de força muscular, de flexibilidade e treinamentos de equilíbrio (CAMACHO, 2018).

O Treinamento de força pode ser definido como um treino de musculação que objetiva o aumento do recrutamento de unidades musculares, no qual, o corpo humano irá solicitar um maior número de fibras dos músculos, em prol de vencer a resistência imposta a ele. O treino de força não pode ser confundido com o treino de hipertrofia, pois este objetiva a ampliação do volume da fibra muscular (CAPRA, 2016).

O treino de força apresenta uma série de benefícios a pessoas com idade igual ou superior a 60 (sessenta) anos, como, por exemplo, a melhoria das capacidades funcionais, como por exemplo, meIhorarias no equilíbrio corporal, na coordenação motora, na agilidade e velocidade e em várias outras atividades que necessitam que o corpo disponha de força muscular (ROCHA, 2016).

O objetivo geral da presente pesquisa consiste em analisar como o treinamento de força pode contribuir no retardo do processo de sarcopenia em pessoas idosas. Dentre os objetivos específicos 
ressaltam-se: Demonstrar a importância do treinamento de força para pessoas com idade igual ou superior a 60 anos; Avaliar o efeito do Treino de Força na capacidade funcional de idosos; Verificar os efeitos do treinamento de força sobre a sarcopenia.

A problemática da pesquisa consiste no seguinte questionamento: De que forma o treinamento de força pode contribuir no retardo do processo de sarcopenia em pessoas idosas?

No que se refere a metodologia a pesquisa pode ser entendida com uma revisão bibliográfica, analisando uma série de fontes sobre a importância dos treinamentos de força em prol de retardar a sarcopenia em pessoas da terceira idade. Na pesquisa foram utilizados um número de 12 artigos científicos que discorrem sobre o Treino de força e sua relação com a sarcopenia por meio da elaboração de um quadro que apresenta o nome do autor, a revista na qual foi publicada, o objetivo da pesquisa, a população participante, a metodologia utilizada, os instrumentos e os resultados de cada pesquisa.

Justifica-se a eleição do presente tema em razão da vulnerabilidade dos idosos devido ao processo natural de envelhecimento, necessitando, dessa forma, de uma maior atenção por parte de uma série de profissionais, como por exemplo profissionais de educação física, os quais podem colaborar em prol do fortalecimento muscular dos idosos e consequentemente proporcionar uma redução da sarcopenia nessa população, evitando uma serie de problemas, como, por exemplo, quedas que podem afetar a integridade física e psíquica dos idosos ou podendo levar ate mesmo a morte.

A pesquisa é pertinente podendo servir de fonte para futuros estudos sobre a relação do treinamento de força com o retardo da sarcopenia em idosos, em especial, para acadêmicos de educação física e outros profissionais da área de saúde, como médico e fisioterapeutas, por exemplo.

\section{METODOLOGIA}

A Para fundamentar a pesquisa referente aos benefícios do treinamento de força no retardo do processo de q em idosos houve a necessidade da realização de revisão sistemática. Segundo Gil (2008) a pesquisa sistemática é construída a partir de pesquisas já desenvolvidas constituídas principalmente de artigos científicos e livros.

Infere-se que a pesquisa pode ser entendida como uma revisão quantitativa e qualitativa, por meio da análise de várias fontes de pesquisa, presentes em sites da internet destinados, principalmente, a pesquisas acadêmicas, ressaltando-se a Scientific Electronic Library OnLine (SCIELO), Medical Literature Analysis and Rterieval System Online (MEDLINE), Literatura Latino-Americana e do Caribe em Ciências da Saúde (LILACS), National Library of Medicine (PubMed), BIREME (Centro Latino Americano e do Caribe de Informação em Ciências da Saúde).

Para uma seleção adequada das fontes a serem utilizadas na fundamentação da presente pesquisa houve uma procura de artigos científicos e livros, além de consultas em sites destinados a pesquisas científicas, sendo utilizados descritores como: Educação física; sarcopenia, treinamento de força.

De acordo com os critérios de inclusão, foram analisados artigos tanto em língua portuguesa, como artigos na língua inglesa, devidamente traduzidos para a língua oficial brasileira, assim como 
só foram considerados artigos publicados entre os anos de 2016 e 2020, procurando desse modo, manter fontes atualizadas sobre o tema em questão.

Para a devida fundamentação da pesquisa houve a necessidade da elaboração de um quadro com as fontes pesquisadas, demonstrando a autoria, o local de publicação, as metodologias que foram aplicados e os resultados logrados por meio da elaboração da pesquisa.

\section{RESULTADOS E DISCUSSÃO}

Considerando como método a revisão sistemática e considerando os descritores já mencionados, foram encontrados trinta e dois (32) artigos nas bases de dados SCIELO (Scientific Eletronic Library On line), LILACS (Literatura Latino Americana e do Caribe em Ciências da Saúde), BIREME (Centro Latino-Americano e do Caribe de Informação em Ciências da Saúde) e PubMed. Os artigos apresentados em mais de uma base de dados foram contabilizados apenas uma vez (Figura 1).

FIGURA 1. Quantidade de artigos conforme a base de dados

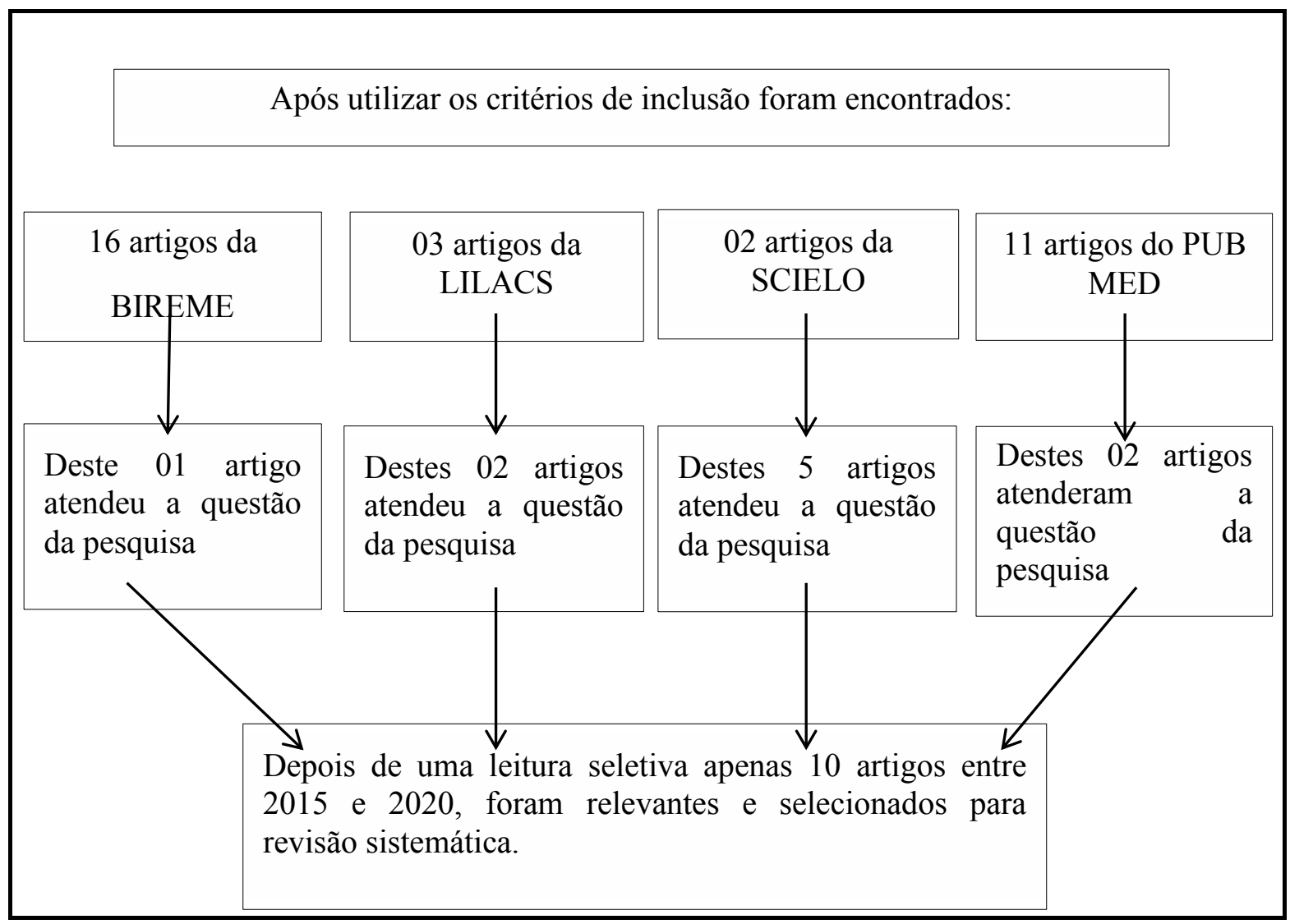

Fonte: Proprio autor (2020). 
QUADRO 1. Benefícios do TF no retardo da sarcopenia em idosos

\begin{tabular}{|c|c|c|c|}
\hline AUTORIA & $\begin{array}{l}\text { BASE DE } \\
\text { DADOS }\end{array}$ & METODOLOGIA & RESULTADOS \\
\hline $\begin{array}{l}\text { CHEN, et. } \\
\text { al. (2017) }\end{array}$ & BIREME & $\begin{array}{l}\text { Os participantes foram designados aleato- } \\
\text { riamente para RT, AT, CT e grupos de con- } \\
\text { trole (CON). Após o treinamento duas vezes } \\
\text { por semana durante } 8 \text { semanas, os partici- } \\
\text { pantes de cada grupo cessou o treinamento } \\
\text { por } 4 \text { semanas antes de ser examinado para } \\
\text { o efeitos de retenção das intervenções de } \\
\text { treinamento. }\end{array}$ & $\begin{array}{l}\text { Adultos mais velhos com obesidade sar- } \\
\text { copênica que se envolveu nas interven- } \\
\text { ções de RT, AT e CT demonstrou aumento } \\
\text { da massa muscular e redução da gordura } \\
\text { total massa e VFA em comparação com } \\
\text { aqueles sem treinamento. O desempe- } \\
\text { nho de força muscular e nível sérico de } \\
\text { IGF-1 em grupos treinados, especialmen- } \\
\text { te no grupo RT, foram superiores para o } \\
\text { grupo de controle. }\end{array}$ \\
\hline $\begin{array}{l}\text { HASSAN } \\
\text { et.al. } \\
(2016)\end{array}$ & LILACS & $\begin{array}{l}\text { Os dados foram coletados no início e após } \\
\text { a intervenção período usando um processo } \\
\text { de avaliação não cego, exceto para o des- } \\
\text { critores de participantes demográficos que } \\
\text { foram coletados apenas em linha de base. } \\
\text { Descritores de participantes foram coleta- } \\
\text { dos da instalação registros, exceto altura } \\
\text { (cm) e peso (kg) que foram medidos usan- } \\
\text { do práticas padrão. Além disso, como uma } \\
\text { medida básica de entrega. A participação EX } \\
\text { foi rastreada ao longo da intervenção como } \\
\text { adesão à sessão. }\end{array}$ & 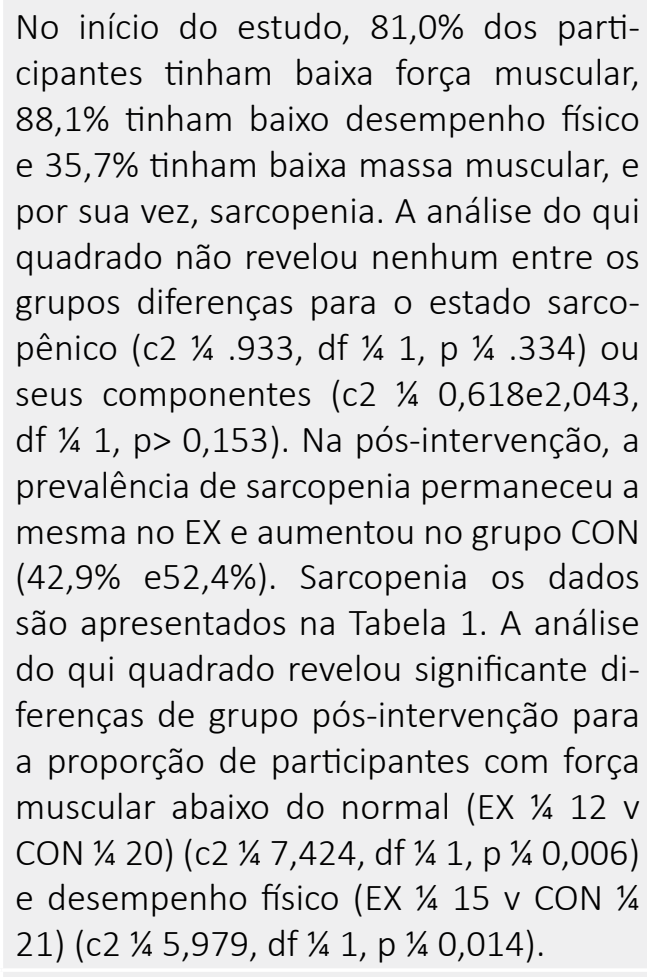 \\
\hline $\begin{array}{l}\text { Chiu et.al } \\
\text { (2018) }\end{array}$ & LILACS & $\begin{array}{l}\text { Este estudo usou um desenho de pesqui- } \\
\text { sa quase experimental. Residentes com } 60 \\
\text { anos ou mais e ter vivido um estilo de vida } \\
\text { sedentário em instalações de LTC nos últi- } \\
\text { mos } 3 \text { meses será elegível para inclusão. }\end{array}$ & $\begin{array}{l}\text { Um total de } 64 \text { entrevistados com SO } \\
\text { completaram o estudo. }\end{array}$ \\
\hline $\begin{array}{l}\text { Liao et.al. } \\
\text { (2017) }\end{array}$ & SCIELO & $\begin{array}{l}\text { Este estudo foi realizado no centro de rea- } \\
\text { bilitação de um hospital universitário e foi } \\
\text { concebido como um ensaio clínico prospec- } \\
\text { tivo e randomizado com análise de intenção } \\
\text { de tratar. }\end{array}$ & $\begin{array}{l}\text { No pós-teste, uma diferença significativa } \\
\text { entre os grupos foi observada na massa } \\
\text { livre de gordura, } M Q \text { e capacidade física } \\
\text { (todos } P<0,05 \text { ); e uma correlação signi- } \\
\text { ficativa foi encontrada entre a alteração } \\
\text { da massa magra da perna e a velocidade } \\
\text { de marcha }(r=0,36 ; P<0,05) \text {. Após } 12 \text { se- } \\
\text { manas de intervenção RET elástica, o EG } \\
\text { teve significativamente menos pacientes } \\
\text { exibindo sarcopenia ( } P<0,05 \text { ) e experi- } \\
\text { mentando dificuldade física ( } P<0,001) \\
\text { do que o CG. }\end{array}$ \\
\hline
\end{tabular}


KIM et. al. (2016)

SCIELO

Mafi et.al. (2018)

SCIELO

Ensaio clínico controlado randomizado supervisionado de 8 semanas

Ensaio clínico randomizado e controlado de quatro braços. Sarcopênicos ou dinapênicos mais velhos adultos foram recrutados para este ensaio. Após a triagem, um total de 112 idosos foram aleatoriamente alocados em quatro grupos; 28 adultos mais velhos cada foram inscritos na resistência combinada grupo de exercícios e suplementação nutricional, grupo de exercícios isolados, grupo de suplementação nutricional grupo sozinho e o grupo controle.

54 idosos (47 mulheres, 7 homens com idade de 72,877 anos) foram aleatoriamente designados para uma das três intervenções: grupo supervisionado (n $=18$ ), individualizado exercícios domiciliares $(n=18)$ e grupo controle $(n=18)$.
Embora o exercício e a nutrição tenham efeitos benéficos nas variáveis individuais do corpo composição, componentes do sangue e função física, melhorias na massa muscular e variáveis.

aumento significativamente maior foi observado na folistatina, relação folistatina / miostatina, leg press e tórax no $R T+E P$ comparando os grupos RT, EP e $P L$, enquanto a miostatina diminuiu significativamente apenas nos grupos RT + EP e RT, no entanto, appMMI e TUG aumentou significativamente em todos os grupos experimentais do que o grupo $\mathrm{PL}$ $(P \leq 0,05)$. Consequentemente, ao comparar os resultados entre três grupos experimentais, a maior melhora foi detectada no grupo RT + EP.

Os participantes do grupo combinado tiveram uma melhora significativamente maior no reto intensidade do eco femoral e torque de extensão do joelho do que nos outros grupos $(P<0,05)$. Além disso, o programa combinado aumentou a massa muscular apendicular em pacientes sarcopênicos adultos mais velhos $(P<0,05)$, mas não em adultos mais veIhos com baixa função física com normal massa muscular.

Os resultados do estudo fornecem dados encorajadores sobre os efeitos do exercício em idosos sarcopênicos. A população sarcopênica é capaz de responder a um programa de exercícios em um ambiente supervisionado ou baseado em casa. Ambas as abordagens podem ser eficazes. No entanto, supervisionado. o exercício em grupo parece ser superior à terapia com exercícios em casa em quase todas as variáveis. Além disso, o programa baseado em grupo induziu melhorias em SMMI, TUG, velocidade de marcha, teste de $4 \mathrm{~m}$, Teste de CS, CC e força do joelho; que foram mantidos por pelo menos 3 meses após o final do programa de exercícios. 


\begin{tabular}{|c|c|c|c|}
\hline $\begin{array}{l}\text { Albino } \\
\text { et.al } \\
\text { (2019) }\end{array}$ & PUBMED & $\begin{array}{l}\text { Este estudo randomizou } 25 \text { idosos com } \\
\text { idade } \geq 65 \text { anos e sarcopenia } 0 \text { progra- } \\
\text { ma de exercícios consistia em } 90 \text { min de } \\
\text { treinamento de força duas vezes por se- } \\
\text { mana durante } 12 \text { semanas. }\end{array}$ & $\begin{array}{l}\text { Houve um consideravel beneficio no pro- } \\
\text { cesso de sarcopenia dos idosos investiga- } \\
\text { dos na presente pesquisa, pois mesmo } \\
\text { nao havendo aumento da força muscular, } \\
\text { houve uma manutençao dessa força. }\end{array}$ \\
\hline $\begin{array}{l}\text { Piastra } \\
\text { et.al. } \\
\text { (2018) }\end{array}$ & PUBMED & $\begin{array}{l}\text { Setenta e dois participantes foram aleato- } \\
\text { riamente atribuídos a dois grupos: o grupo } \\
\text { de treinamento de reforço muscular (RESIS- } \\
\text { TÊNCIA) ( } n=35 ; 69,9 \pm 2,7 \text { anos) e o postu- } \\
\text { ral grupo de treinamento (POSTURAL) ( } n= \\
37 ; 70,0 \pm 2,8 \text { anos). Composição corporal, } \\
\text { massa muscular, índice de massa muscu- } \\
\text { lar esquelética (SMI) e a força de preensão } \\
\text { manual (FPM) foi avaliada para avaliação da } \\
\text { sarcopenia, enquanto o caminho de osci- } \\
\text { lação, área de oscilação, tempo de perma- } \\
\text { nência e distância espacial foram avaliados } \\
\text { para avaliação do equilíbrio estático. Ses- } \\
\text { senta e seis participantes completaram o } \\
\text { estudo (grupo RESISTÊNCIA: } n=33 \text {; POSTU- } \\
\text { RAL grupo: } n=33 \text { ). }\end{array}$ & $\begin{array}{l}\text { De maneira geral, os resultados deste } \\
\text { estudo sugerem que o programa APA ba- } \\
\text { seado no reforço muscular aplicado em } \\
\text { mulheres idosas sarcopênicas modera- } \\
\text { das foi capaz de melhorar significativa- } \\
\text { mente a massa muscular e a força mus- } \\
\text { cular, e também foi mais eficaz do que o } \\
\text { protocolo postural aplicado na determi- } \\
\text { nação de efeitos positivos no equilíbrio } \\
\text { estático. }\end{array}$ \\
\hline
\end{tabular}

Fonte: Próprio autor (2020)

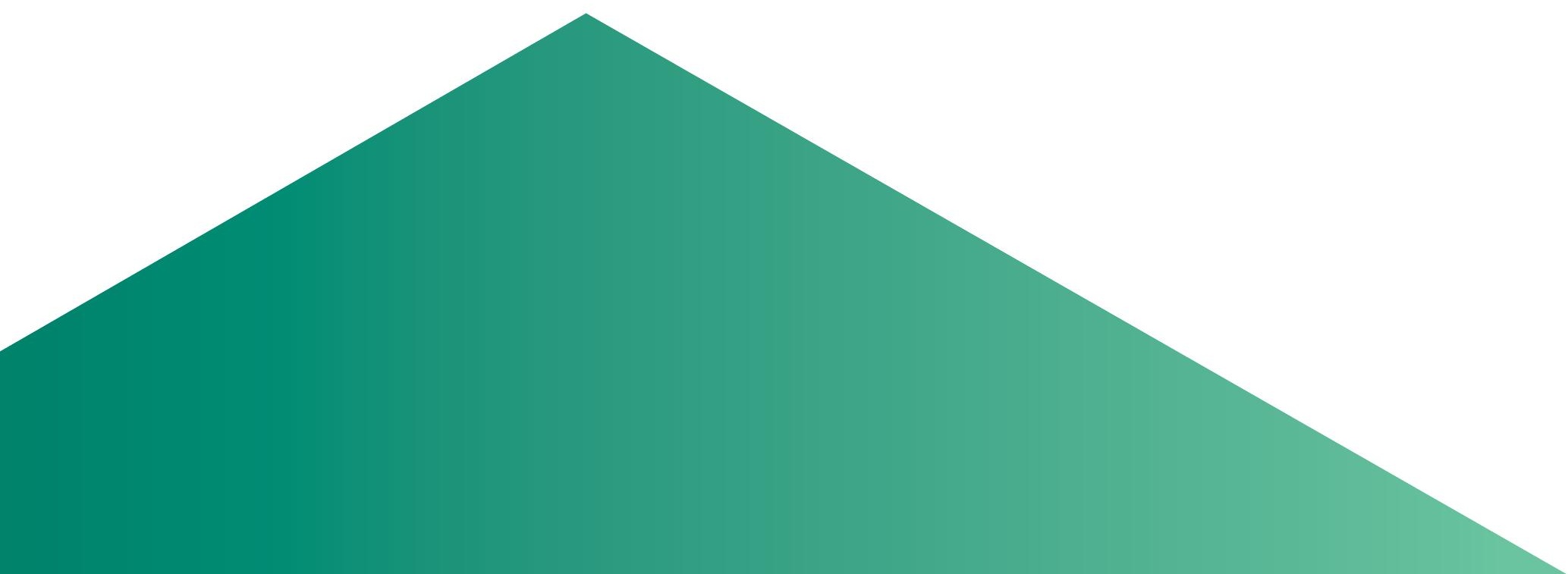


Conforme a pesquisa foi possível identificar, por meio da literatura que os resultados da atuação do treinamento de força no retardo da sarcopenia de idosos, são significativos. Os idosos com obesidade sarcopênica demonstram por meio do treianmento de força um aumento da massa muscular e redução total massa gorda e AGV (CHIEN et. al, 2017).

Saienta-se que por meio do treinamento de força e de treinamento de resistência idosos podem ser capazes de interromper sua transição para sarcopenia, além de aumentar sua força muscular e reduzir seu IMC. Embora nem sempre ocorra ganhos significativos de massa muscular, isso não é incomum, mas como o principal componente da síndrome geriátrica sarcopenia, intervenções futuras precisam resolver isso se a prevalência sarcopênica deve ser reduzida nesse cenário.

O reforço muscular aplicado, principalmente, em mulheres idosas sarcopênicas moderadas foi capaz de melhorar significativamente a massa muscular e também a força muscular, e sendo considerado mais eficaz do que o protocolo postural aplicado na determinação de efeitos positivos no equilíbrio estático (PIASTRA, et. al, 2018).

O treinamento de força pode minimizar ou retardar o processo de sarcopenia para obter significantes respostas neuromusculares (hipertrofiamuscular e força muscular), por meio do aumento da capacidade contrátil dos músculos esqueléticos. O treinamento de força é um fator extremamente atuante para evitar quedas nas situações de desequilíbrio do corpo, pelo aumento da massa magra e força muscular. Sugere-se que esse tipo de treinamento, por no mínimo duas vezes por semana, com cargas de aproximadamente $70 \%$ da carga máxima e com ênfase maior aos membros inferiores, produz melhoras significativas no equilíbrio corporal em idosos (ALBINO, 2015).

Os resultados do estudo fornecem dados encorajadores sobre os efeitos do treinamento de força em idosos sarcopênicos. A população sarcopênica é capaz de responder a um programa de exercícios em um ambiente supervisionado ou baseado em casa. Ambas as abordagens podem ser eficazes. No entanto, supervisionado o exercício em grupo parece ser superior à terapia com exercícios em casa em quase todas as variáveis. Além disso, o programa baseado em grupo induziu melhorias em SMMI, TUG, velocidade de marcha, teste de $4 \mathrm{~m}$, Teste de CS, CC e força do joelho; que foram mantidos por pelo menos 3 meses após o final do programa de exercícios. 


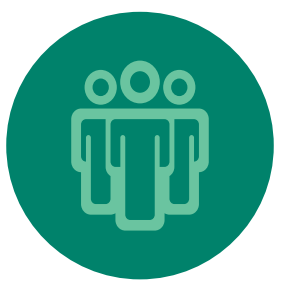

\section{CONCLUSÃO}

Por meio da presente pesquisa foi possível constatar que o treinamento de força muscular é capaz de trazer uma serie de benefícios para a saúde de pessoas da terceira idade, como por exemplo a meIhora do equilíbrio e o retardo do processo de sarcopenia.

O treinamento de força é capaz de minimizar ou causar um retardo no processo de sarcopenia para obter respostas neuromusculares, ou seja, hipertrofiamuscular e força muscular, por meio do aumento da capacidade contrátil dos músculos esqueléticos. O treinamento de força tem sido apontado como um fator atuante para evitar quedas nas situações de desequilíbrio do corpo, pelo aumento da massa magra e da força muscular.

Além disso constatou-se na pesquisa que a atuação do treinamento de força no retardo da sarcopenia de idosos, são significativos, principalmente pessoas da terceira idade que apresentam obesidade sarcopênica que demonstram por meio do treianmento de força um aumento da massa muscular e redução total massa gorda e AGV.

Embora esteja presente na literatura várioas estudos sobre a presente temática, salienta-se que ainda são necessárias a elaboração de mais pesquisas referentes ao processo de retardo da sarcopenia por meio das diversas modalidades de treinamento de força. 


\section{REFERÊNCIAS}

Albino, Igna Luciara. Influência do treinamento de força muscular e de flexibilidadearticular sobre o equilíbrio corporal em idosas. REV. BRAS. GERIATR. GERONTOL., RIODE JANEIRO, 2015.

CAMACHO, R. et al. Efeitos de 12 semanas de TF e ginástica em circuito na autonomia funcional em idosas. Rev. Bras. de Presc. e Fisiol. do Exerc.,São Paulo,v. 12, n. 72, p. 112-119, jan-fev/2018.

CAPRA, D. et al. Influência do treinamento de força em programas de emagrecimento. Arch. Health Invest., v. 5, n. 1, p. 1-7, jan-fev/2016.

Chien, Hung-Ting et. al. Effects of Different Types of Exercise on Body Composition, Muscle Strength, and IGF-1 in the Elderly with Sarcopenic Obesity. JAGS, 2017

Mijnarends DM, Schols JM, Meijers JM, Tan FE, Verlaan S, Luiking YC, et al. Instruments to assess sarcopenia and physical frailty in older people living in a community (care) setting: similarities and discrepancies. J Am Med Dir Assoc. 2015.

Hassan, PHD et. al. Impact of resistance training on sarcopenia in nursing care facilities: A pilot study. Article history. Geriatric Nursing. Received 30 August 2015

IBGE (Instituto Brasileiro de Geografia e Estatística). Estudos e Pesquisas, Informação Demográfica e Socioeconômicas: Síntese de Indicadores Sociais 2018. n.19, Rio de Janeiro: 2019.

Izquierdo M, Cadore EL. Muscle power training in the institutionalized frail: a new approach to counteracting functional declines and very late-life disability. Curr Med Res Opin. 2014

Pagotto V. Applicability and agreement of different diagnostic criteria for sarcopenia estimation in the elderly. Arch Gerontol Geriatr 2014.

Steffl M. Alcohol consumption as a risk factor for sarcopenia-a meta-analysis. BMC Geriatrics 2016;

SIQUEIRA, R. L. Velhice: algumas considerações teóricas e conceituais. Ciência. Saúde Coletiva, Rio de Janeiro, v.7, n.4, p. 899-906, 2015.

Tezekoura, M. The Effects of Group and Home-Based Exercise Programs in Elderly with Sarcopenia: A Randomized Controlled Trial. J. Clin. Med. 2018, 7, 480; doi:10.3390/jcm7120480. 